\title{
Dependency Analysis for Control Flow Cycles in Reactive Communicating Processes
}

\author{
Stefan Leue ${ }^{1}$, Alin Ştefănescu ${ }^{2, \star}$, and Wei Wei ${ }^{1}$ \\ ${ }^{1}$ Department of Computer and Information Science \\ University of Konstanz \\ D-78457 Konstanz, Germany \\ \{Stefan.Leue, Wei. Wei\}@uni-konstanz.de \\ ${ }^{2}$ SAP Research CEC Darmstadt \\ Bleichstr. 8, D-64283 Darmstadt, Germany \\ alin.stefanescu@sap.com
}

\begin{abstract}
The execution of a reactive system amounts to the repetitions of executions of control flow cycles in the component processes of the system. The way in which cycle executions are combined is not arbitrary since cycles may depend on or exclude one another. We believe that the information of such dependencies is important to the design, understanding, and verification of reactive systems. In this paper, we formally define the concept of a cycle dependency, and propose several static analysis methods to discover such dependencies. We have implemented several strategies for computing cycle dependencies and compared their performance with realistic models of considerable size. It is also shown how the detection of accurate dependencies is used to improve a livelock freedom analysis that we developed previously.
\end{abstract}

\section{Introduction}

The main purpose of a concurrent reactive system is to maintain an ongoing interaction with its environment [15]. The execution of the system is therefore expected to last forever. Since each component process in the system has a finite control structure, any infinite execution of the system is essentially an infinite repetition of a certain set of control flow cycles in the concurrent processes that form the system. The way in which cycle executions are combined is certainly not arbitrary. For instance, the repetition of one cycle may rely on the repetitions of some other cycles; and the execution of one cycle may also eliminate the possibility of executing some other cycles.

We believe that the information of such cycle dependencies is important to the design, understanding, and verification of reactive systems. As one example, the knowledge of cycle dependencies may reveal potential design errors. In a reactive system, let us suppose that the repetition of a control flow cycle $C$ relies on the repetitions of other cycles. When we expect $C$ to be executed infinitely often,

\footnotetext{
* The work was done while this author was working at the University of Konstanz.
} 
we may want to check statically whether there is any other cycle in the system on which $C$ relies. The fact that no such cycles can actually be found hints at an incompleteness in the design or implementation of the system.

The knowledge of cycle dependencies is also useful in the verification of concurrent reactive systems. In our precursory work, we proposed an efficient system verification framework based on integer linear program (ILP) solving [13,12]. Our verification methods abstract the original verification problem into an ILP problem that describes a necessary condition for the violation of the property under scrutiny. Any solution to the ILP problem corresponds to a counterexample in the form of a set of cycles. A counterexample is spurious if it is impossible to repeat the cycles in the counterexample forever without other cycles also being repeated infinitely often. Consequently, the dependency among cycles stands at the very core of the refinement procedure based on the detection of spurious counterexamples in $[14,12]$.

The central contribution of this paper is a formal framework capturing a notion of dependency between the control flow cycles of the concurrent processes. We also inspect different causes of dependencies, and develop techniques for discovering dependencies with respect to each cause. In this paper we choose Promela [9] as modeling language for the systems that we analyze. This choice is motivated by convenience since a large number of Promela models are available in the public domain [21] and some of the features of the SPIN tool environment, which interprets Promela, greatly facilitate our static analysis. We conjecture that applying our analysis ideas to other modeling and programming languages based on communicating finite state machines, such as UML-RT, could easily be accomplished.

Related Work. To the best of our knowledge, there is currently no work addressing control flow cycle dependencies. Control flow graphs of general programs were extensively studied in the area of static program analysis [20] with applications, e.g., in the area of compiler optimization. Slicing of programs $[25,7,18,23]$ checks dependences between statements but not cycles. The "may happen in parallel" [19] and "non-concurrency" [16] analyses also consider dependences between statements. Finally, the INCA verification framework [4,24] studies the relation between acyclic paths and control flow cycles but not relations among cycles. Moreover, the above techniques are applied to either sequential programs or synchronous communication settings, while we also address an asynchronous setting where exchanging messages via buffers is the dominant way of communication.

Structure of the Paper. Section 2 introduces the Promela modeling language, define cycles and some related concepts. Section 3 defines the concept of cycle dependencies. We propose in Sections 4 and 5 several static analysis methods for cycle dependency discovery. Section 6 briefly shows how the discovery of cycle dependencies can help improve the precision of a livelock freedom test. Section 7 reports the experimental results, before Section 8 concludes the paper. All the proofs of the theoretical results of the paper can be found in Appendix A. 


\section{Preliminaries}

Promela. Promela is the input language of the SPIN explicit state model checker [9]. It has been successfully used for the modeling and analysis of many concurrent systems $[10,6]$. The Promela language supports asynchronous communication as well as synchronous rendez-vous communication and synchronization via shared variables. The subset of the Promela language that we consider includes the definition of concurrently running processes ("proctype"), communication channels ("chan" declarations), message sending ("!") and receiving ("?"), assignments, condition statements, nondeterministic branching ("if . . $f i$ "), looping ("do ... od"), and arithmetics. For the sake of simplicity we do not consider arrays and structured data types in this paper.
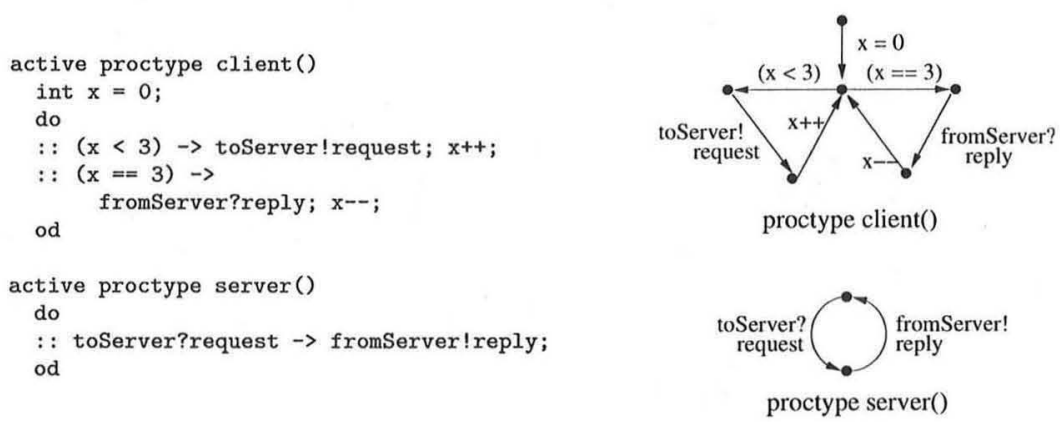

Fig. 1. An example Promela model and its control flow graphs

Figure 1 shows a simple Promela model consisting of two processes: client and server, whose behavior is described by the sequential Promela code within the respective proctype definition. The process client may send a request message to the buffer toServer if $x<3$ (Line 4). Otherwise, it waits until a reply message is available in the buffer fromserver and then receives the message (Line 6). A condition statement such as $(x<3)$ is a boolean expression enclosed in parentheses, which acts as a guard to the subsequent statements. It is executable if and only if the enclosed expression evaluates to true. We can construct a control flow graph from each of the proctype definitions (see Figure 1). Each transition corresponds to one statement in the code, and its source state and target state respectively denote the control points before and after the execution of the statement.

Control flow cycles. We define a control flow cycle (or simply cycle) in a control flow graph as a sequence of consecutive transitions in the graph such that the source state of the first transition in the sequence is the same as the target state of the last transition. A cycle is elementary (or simple) if no two transitions in the defining sequence of the cycle have a same source state. Informally, an elementary cycle cannot be decomposed further into smaller cycles. In the control flow graph of the process client in Figure 1, there are two elementary cycles. 
Even though a finite control flow graph may contain infinitely many cycles, the number of elementary cycles is always finite and in the worst case exponential in the number of transitions. Since any non-elementary cycle can be decomposed into elementary cycles, our analysis considers only elementary cycles. Unless otherwise specified, all the cycles mentioned in the following are elementary.

If two cycles share states, then they are neighbors of each other. Any such shared state is an exit state of the cycles that contain it, because one can exit one cycle at that state and enter another cycle. The two cycles in the process client in Figure 1 are neighbors sharing one exit state.

Cycle executions. An infinite run of a Promela model amounts to the repeated executions of cycles in some processes of the model. For an infinite run $r$ of a Promela model, let $r / p$ denote the projection of $r$ on the set of transitions in a process $p$. Thus, $r / p$ corresponds to the local execution of $p$ in $r$. Any $r / p$ can be decomposed into two parts: (1) an acyclic path from the initial state, and (2) repeated executions of cycles. Given a cycle $c$ in $p$, one execution of $c$ in $r / p$ may be interrupted by the executions of other cycles in $p$ : Some part of $c$ is executed until some exit state $s$ is reached where it starts to execute other cycles. The execution of $c$ is later resumed from $s$ after the executions of those interrupting cycles are completed. Since $r$ is an infinite run, at least one cycle in the model is repeated infinitely often. We denote by $\operatorname{IRC}(r)$ (infinitely repeated cycles) the set of cycles that are executed infinitely often in $r$. For a process $p$, $\operatorname{IRC}(\mathrm{r} / \mathrm{p})$ is the subset of $\operatorname{IRC}(r)$ consisting of only cycles in $p$. It is easy to see that $\operatorname{IRC}(r / p)$ is either empty or forms a strongly connected subgraph of the control flow graph of $p$.

\section{Cycle Dependencies}

We now define the concept of cycle dependencies. Intuitively, a cycle $c$ depends on a set of cycles $S$ if the infinite execution of $c$ must be accompanied by the infinite executions of some cycles in $S$.

Definition 1. Given a Promela model, a cycle $c$ and a set of cycles $S$ in the model, we call the pair $(c, S)$ a cycle dependency if they satisfy the following conditions: a) $c \notin S$; and b) for any infinite run $r$ of the model where $c \in \operatorname{IRC}(r)$, there exists a cycle $c^{\prime} \in S$ such that $c^{\prime} \in I R C(r)$. In this case, we say that $c$ depends on $S$.

In the above definition, if all the cycles in $S$ are in the same process as $c$ is, then $(c, S)$ is a local dependency. Otherwise, $(c, S)$ is a global dependency. Moreover, if $c$ does not depend on any subset of $S$, then we say that $(c, S)$ is a minimal dependency. In the model in Figure 1, we denote by $c_{l}$ (resp. $c_{r}$ ) the left (resp. right) cycle in the process client and by $c_{s}$ the only cycle in the process server. $\left(c_{r},\left\{c_{l}, c_{s}\right\}\right)$ is a cycle dependency, while $\left(c_{r},\left\{c_{l}\right\}\right)$ and $\left(c_{r},\left\{c_{s}\right\}\right)$ are two minimal cycle dependencies. In particular, $\left(c_{r},\left\{c_{l}\right\}\right)$ is a local dependency, and $\left(c_{r},\left\{c_{s}\right\}\right)$ is a global dependency. 
If we interpret all message buffers in a Promela model to have only finite capacities, then the Promela model possesses a finite global state space. In this case, we show as follows that it is decidable whether $(c, S)$ is a cycle dependency: We construct the global state space for the model and then look for any elementary of non-elementary cycle in the global state space that contains $c$ but no cycles from $S$. If no such global cycles exist, then $(c, S)$ is a cycle dependency. However, we are more interested in infinite state models. If we assume that buffers in Promela models have infinite capacities and variables may have infinite domains such as integer variables, then a Promela model may have an infinite global state space, for which we show in the following theorem that the above problem becomes undecidable.

Theorem 1. Given a cycle $c$ and a set $S$ of cycles, it is undecidable in general whether $(c, S)$ is a cycle dependency.

\subsection{The Causes of Cycle Dependencies}

The root cause for cycle dependencies lies in the executability of Promela statements. Given a cycle, if the executability of every statement along the cycle is unconditional, then the cycle can be repeated without interruption forever once the cycle is entered. Such a cycle does not depend on any other cycles. On the contrary, consider a cycle $c$ that contains a statement $s$ whose executability is conditional. If $s$ cannot be continuously enabled forever by only repeating $c$, then some other cycles need to be executed in order to re-enable $s$ by, e.g., modifying the values of some variables, sending a message etc. In Promela there are two kinds of statements with conditional executability: condition statements and message receiving statements, when we take the assumption that message buffers have unbounded capacities and message sending statements are therefore always enabled. In the following we explain how cycle dependencies may be imposed by these two kinds of statements.

Condition statements. Consider the right cycle $c_{r}$ in the process client in Figure 1. $c_{r}$ contains a condition statement $(\mathrm{x}==3$ ). The condition $x=3$ cannot remain true after $c_{r}$ is executed because $x$ is decremented by 1 in the cycle. Then, $c_{r}$ can be repeated infinitely often only if the left cycle $c_{l}$ is also repeated infinitely often to modify the value of $x$ such that $x$ can always acquire the value 3 again. This is one example that a cycle is terminating on a condition statement along the cycle. Since we focus on discovering cycle dependencies in this paper, it is out of scope how to determine whether a cycle is terminating, which is a well-known undecidable problem. In [14] we proposed an incomplete procedure to prove termination for control flow cycles. There are also many existing techniques $[22,3,5,1]$ to prove termination for certain kinds of loops in programs, which can be adapted to prove termination for control flow cycles. In Section 4 we will show how to determine cycle dependencies from a condition statement on which a cycle is terminating. 
Message receiving statements. The above mentioned cycle $c_{r}$ contains a message receiving statement fromServer?reply. Thus, the cycle $c_{s}$ sending reply messages has to be repeated infinitely often when $c_{r}$ is to be repeated infinitely often. In Section 5 we will present a method to determine cycle dependencies from message receiving statements, which are usually global dependencies.

\section{Discovering Dependencies from Condition Statements}

We show some types of cycle dependencies imposed by condition statements on which a cycle is terminating. In order to derive them, we need to discriminate between different ways in which the variables in a condition statement are modified in the cycle. A variable is local if its value can be referenced and modified only by one process. Otherwise, it is a global variable. However, the runtime value of a local variable may still depend on the executions of other processes. For instance, given a local variable $x$, if there is an assignment $\mathrm{x}=e(y)$ where $e$ is an arithmetic expression containing a global variable $y$, then the runtime value of $x$ may depend on how $y$ is modified in other processes.

Definition 2. For a cycle $c$ and a variable $x, x$ is globally modified in $c$ if one of the following is satisfied: a) $x$ is global, or $b$ ) there is a message receiving statement $\mathrm{b}$ ? $\mathrm{msg}\left(x_{1}, \ldots, x_{n}\right)$ in $\mathrm{c}$ where $x$ is some $x_{i}$, or $\left.c\right)$ there is an assignment $\mathrm{x}=e(y)$ in $c$ where $y$ is globally modified in $c$. Otherwise, $x$ is locally modified in $c$.

Note that in the above definition we disregard the dependency of the runtime value of a local variable on a condition statement. The reason is that a control flow cycle contains only one branch of a condition statement. Therefore, the impact of the condition statement is fixed in the cycle. Note that we are only interested in how a variable is modified inside a particular cycle when the cycle is repeated without interruption.

For a boolean condition $B$ in a cycle $c$, we denote by $\operatorname{var}(B)$ the set of variables occurring in $B$. If all the variables in $\operatorname{var}(B)$ are locally modified in $c$, then $B$ is a locally determined condition. Otherwise, it is globally determined. In Subsection 4.1 and 4.2, we show how to determine cycle dependencies from these two kinds of conditions.

\subsection{Locally Determined Conditions}

First, we can easily see that, if a cycle is terminating on a locally determined condition, then it depends on some of the cycles in the same process for an infinite number of executions. In particular, the cycle must depend on one of its neighbors.

Proposition 1. Given a cycle $c$ in a process $p$ such that $c$ is terminating on a locally determined condition $B$, if $c$ is repeated infinitely often in a run $r$, then one of the neighbors of $c$ is also repeated infinitely often in $r$.

Let $C_{p}$ denote the set of cycles in $p$, and $N_{c}$ denote the set of the neighbors of $c$. The above discussion gives two cycle dependencies, namely $\left(c, C_{p}-\{c\}\right)$ and 
$\left(c, N_{c}\right)$. The cycle $\left(c, C_{p}-\{c\}\right)$ is usually coarse because not necessarily all the cycles in $p$ contribute to the re-satisfaction of $B$. In the following, we propose several methods to refine the dependency $\left(c, C_{p}-\{c\}\right)$.

Refinement 1. In general, it is impossible to determine which cycles make a contribution to the re-satisfaction of the condition $B$. We define $E_{c}(B)$ as the set of variables occurring in $c$ such that at least one of the variables in $E_{c}(B)$ must be modified in order to make $B$ true again. The set $E_{c}(B)$ subsumes but not necessarily equals $\operatorname{var}(B)$. In the example in Figure 2, an infinite number of repetitions of the left cycle relies on an infinite number of repetitions of the right one that resets the value of $y$. However, the enabling condition of the left cycle contains only the variable $x$ which is not modified by the right cycle. We propose the following recursive method to compute $E_{c}(B)$. A variable $v$ is in $E_{c}(B)$ if one of the following is satisfied: a) $v \in \operatorname{var}(B)$, or b) there is an assignment $v^{\prime}=$ $e(v)$ in $c$ such that $v^{\prime} \in E_{c}(B)$. For a set $S$ of variables, we denote by $M C_{p}(S)$ the set of cycles in $p$ which modify at least one variable in $S$. We obtain a finer dependency $\left(c, M C_{p}\left(E_{c}(B)\right)-\{c\}\right)$ by disregarding all cycles that do not modify any variables in $E_{c}(B)$.
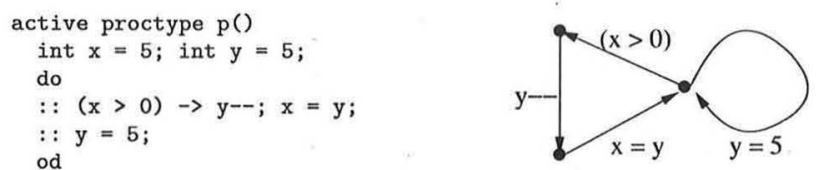

Fig. 2. An example Promela model and its control flow graph

Refinement 2. The above cycle dependency may still be coarse. Consider the control flow graph in Figure 3. Note that, whenever leaving $C 1$ to execute $C 3$, $C 2$ is always executed. So, in any run in which $C 1$ is repeated infinitely often, no matter whether $C 3$ is repeated infinitely often or not, $C 2$ is always repeated infinitely often. Based on this observation we can refine the cycle dependency $(C 1,\{C 2, C 3\})$ by safely removing $C 3$. The above simple example leads to the following definition.
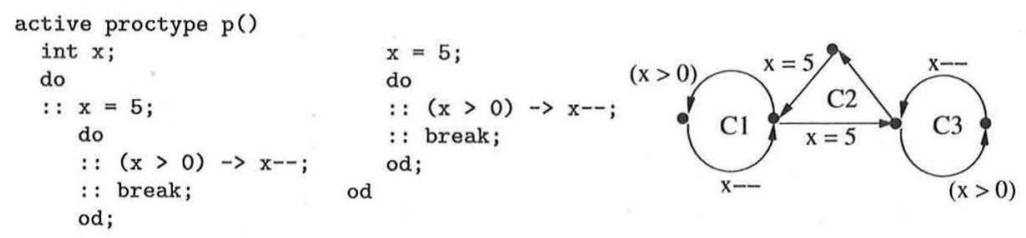

Fig. 3. An example Promela Model and its control flow graph

Definition 3. Given a cycle $c$ in a process $p$ such that $c$ is terminating on a locally determined condition $B$, and a cycle $c^{\prime} \in M C_{p}\left(E_{c}(B)\right)$ such that $c$ and $c^{\prime}$ are reachable from each other, $c^{\prime}$ is preemptive with respect to $c$ and $B$ if there 
exist one exit state $s$ in $c$ and one exit state $s^{\prime}$ in $c^{\prime}$ such that a) there is an acyclic path from $s$ to $s^{\prime}$ that does not modify any variables in $E_{c}(B)$, and $b$ ) there is an acyclic path from $s^{\prime}$ to $s$ that does not modify any variables in $E_{c}(B)$. Otherwise, $c^{\prime}$ is preempted.

In the previous example, $C 2$ is preemptive and $C 3$ is preempted. It is easy to prove that, on the way from any cycle $c$ to execute one of its preempted cycles and then back to $c$, at least one preemptive cycle must be executed. We can therefore refine the cycle dependency $\left(c, M C_{p}\left(E_{c}(B)\right)-\{c\}\right)$ by removing all the preempted cycles from $\left(M C_{p}\left(E_{c}(B)\right)-\{c\}\right)$.

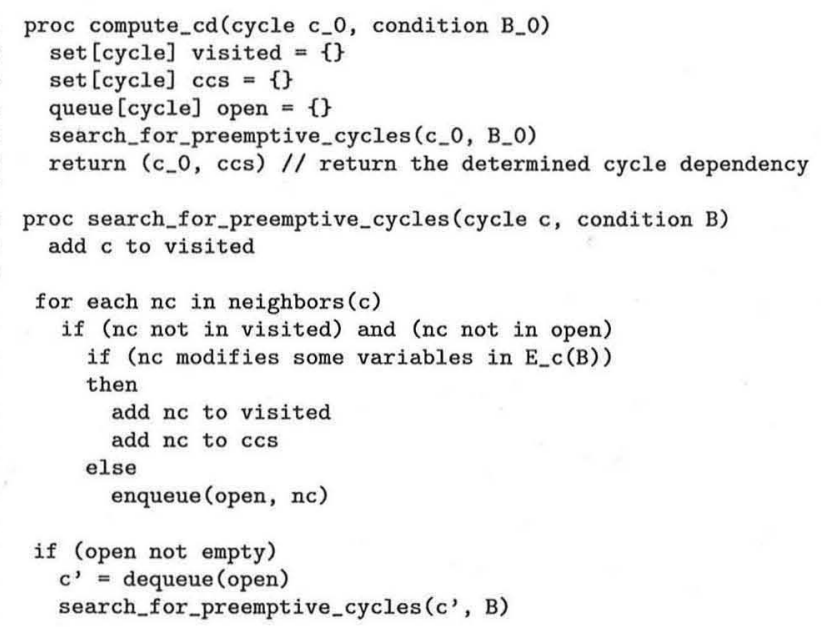

Fig. 4. An algorithm to determine cycle dependencies from locally determined conditions

Whereas Definition 3 can be used to determine whether a cycle is preempted, Figure 4.1 gives an efficient algorithm to collect preemptive cycles during the computation of cycle dependencies. In a Breadth First Search manner, the algorithm visits each cycle at most once, and thus is linear in the number of cycles. This is a generalization of the so-called "next door" strategy first mentioned in [12]. In Appendix A.3 the termination and soundness of the algorithm are proved.

\subsection{Globally Determined Conditions}

If a cycle is terminating on a globally determined condition, then it may not only depend on cycles in the same process, because cycles in other concurrent processes can possibly influence the runtime value of the condition. This can be illustrated in the example in Figure 5. The cycle in Process $p$ is actually the only cycle in $p$, and it depends on the cycle in $q$. We will not consider any globally determined condition whose value is influenced by a message receiving statement, which will be discussed in the next section. 

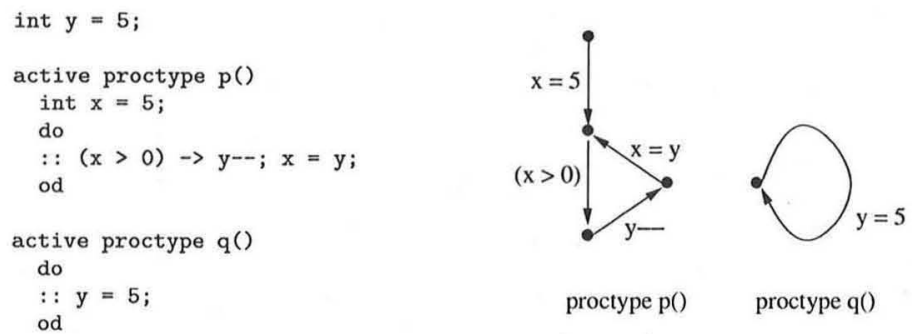

Fig. 5. An example Promela model and its control flow graphs

For a Promela model $M$, we denote by $\operatorname{proc}(M)$ the set of processes in $M$. Suppose a cycle $c$ in a process in $M$ such that $c$ is terminating on a globally determined condition $B$. We can easily derive that $c$ depends on $\left(\bigcup_{q \in \operatorname{proc}(M)} M C_{q}\right.$ $\left.\left(E_{c}(B)\right)-\{c\}\right)$. We may refine this cycle dependency by using the algorithm in Figure 4.1 to rule out all the preempted cycles in $M C_{p}\left(E_{c}(B)\right)$ if $c$ is in the process $p$.

\section{Discovering Dependencies from Message Receiving Statements}

When a cycle $c$ contains a message receiving statement $\mathrm{b}$ ?msg $\left(x_{1}, \ldots, x_{n}\right)$, it needs an infinite number of msg messages to be repeated infinitely often. Consequently, $c$ depends on some cycles that send such messages. Let $S C_{b, m s g}$ be the set of the cycles sending messages msg to $b$. If $c \notin S C_{b, m s g}$, then $\left(c, S C_{b, m s g}\right)$ is cycle dependency. In the remainder of the section, we assume that a cycle never receives messages sent by itself.

A cycle that receives messages may contain a condition statement in which the condition contains some variables used to store components of received messages. Usually, the cycle can be executed only if the received message contains such components that make the condition true. Consider a cycle that contains a message receiving statement $s_{1}$ and a condition statement $s_{2}$ such that the condition in $s_{2}$ contains variables used in $s_{1}$. The following pattern for $s_{1}$ and $s_{2}$ are observed in most real life Promela models: (1) all the variables in $s_{1}$ are local; (2) the condition in $s_{2}$ contains only variables used in $s_{1}$; (3) no variable in the condition is modified between $s_{1}$ and $s_{2}$ in the cycle. We call such a condition a message determined condition. Figure 6 shows two processes GIOPClient and GIOPAgent. In the control flow graph of GIOPClient, there is a cycle depicted using only solid lines that contains a message determined condition reply_status $=4$. We show in the remainder of the section how to derive cycle dependencies from such a message determined condition.

In Figure 6, let $c_{1}$ denote the solid-lined cycle in Process GIOPClient, and $c_{2}$ and $c_{3}$ denote the cycles that respectively assign 4 and 5 to $r s$ in Process GIOPAgent. We have a dependency $\left(c_{1}, S C_{\text {toClientL, Reply }}\right)$ and both $c_{2}$ and $c_{3}$ are 


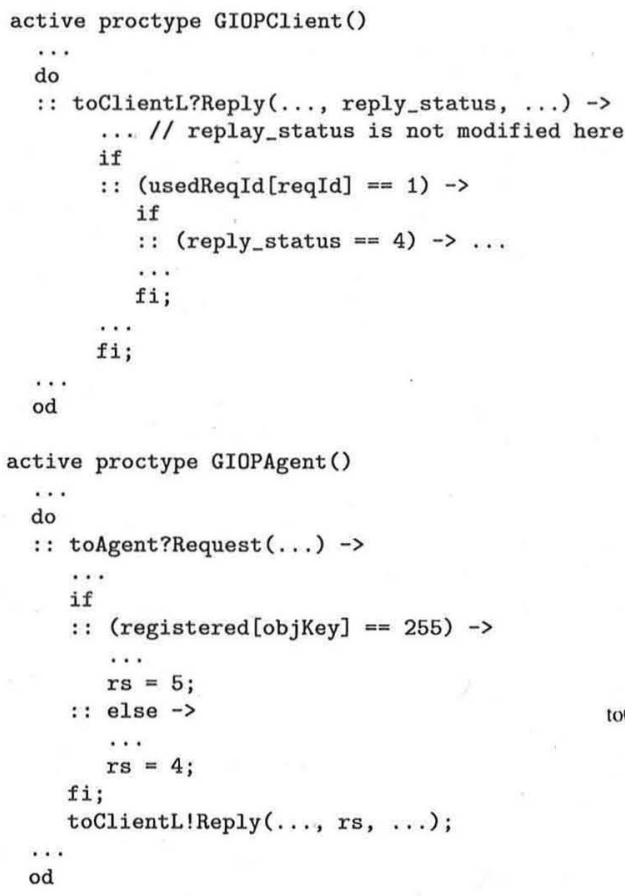

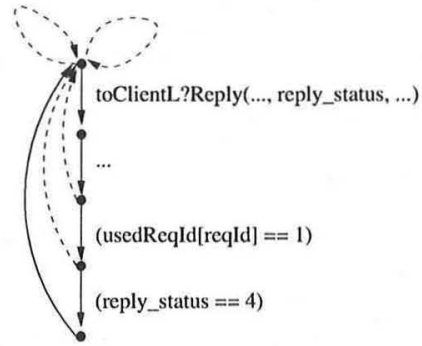

proctype GIOPClient()

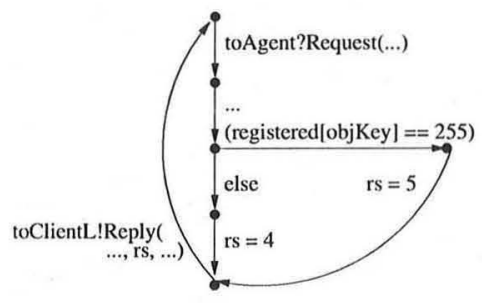

proctype GIOPAgent()

Fig. 6. An excerpt from a Promela model for CORBA GIOP [10]

in $S C_{\text {to ClientL, Reply. }}$. However, this dependency is coarse because not necessarily every cycle in $S C_{\text {toClient } L, \text { Reply }}$ may send a Reply message to make reply_status = 4 true in $c_{1}$. As an example, $c_{3}$ assigns 5 to $r s$ whose value is passed to reply_status in $c_{1}$ through message passing. Thus, $c_{3}$ cannot make reply_status $=4$ true, and it can be safely removed from $S C_{\text {toClient } L, \text { Reply }}$ to obtain a finer dependency. Now the question is how to determine which cycle cannot send messages to make reply_status $=4$ true.

First, we need to determine which kind of Reply messages must be received by $c_{1}$ to make reply_status $=4$ true. More precisely, we need to know which condition must be satisfied by the components of such a message. According to the definition of message determined conditions, reply_status is not modified in $c_{1}$ between the message receiving statement and (reply_status $==4$ ). However, after a message is received, reply_status may still be modified before (reply_status $==4$ ) is reached. This is because the execution of $c_{1}$ can be interrupted, e.g., at the source state of the transition corresponding to (usedReqId [reqId] ==1). Then, when the execution of $c_{1}$ is resumed, reply_status may be already modified by other cycles. However, in this concrete example, if $c_{1}$ is interrupted, then before $c_{1}$ is resumed the last completed interrupting cycle always receives a Reply message. Moreover, this message contains a component whose value is passed to reply_status. The value of reply_status is afterward unchanged before reaching the message determined condition. This is because $c_{1}$ and its neighbors satisfy the 
following structural property named fastened cycles: Given a cycle $c$ that contains a message receiving statement $s_{1}$ and a condition statement $s_{2}$, we denote by $t_{1}$ the transition corresponding to $s_{1}$, by $t_{2}$ the transition corresponding to $s_{2}$, and by $p$ the path from the source state of $t_{1}$ to the source state of $t_{2}$. For each neighbor $c^{\prime}$ of $c$, if $c^{\prime}$ and $c$ contain a common state $s$ within $p$, then $c^{\prime}$ contains also the path in $c$ from the source state of $t_{1}$ to $s$. The pattern in the fastened cycles property results from nested if statements inside do loops which are a common control structure of concurrent processes in an asynchronous reactive system.

Proposition 2. Let $c$ be a cycle that contains a condition statement $(B)$ in which the condition $B$ is determined by messages received via the statement $\mathrm{b}$ ? $\operatorname{msg}\left(x_{1}, \ldots, x_{n}\right)$ in $c$. If the fastened cycles property is satisfied by $c$ and all of its neighbors, then one execution of $c$ needs a $\operatorname{msg}\left(d_{1}, \ldots, d_{n}\right)$ message such that $B\left[x_{i} \leftarrow d_{i}\right]^{1}$ is true.

Using Proposition 2, if we can determine that the execution of $c$ requires a message msg $\left(d_{1}, \ldots, d_{n}\right)$ such that $B\left[x_{i} \leftarrow d_{i}\right]$ is true, then we can use the following method to determine whether a cycle $c^{\prime}$ may not send such a message. Given a cycle $c^{\prime}$ that contains a message sending statement $\mathrm{b} ! \mathrm{msg}\left(d_{1}, \ldots, d_{n}\right)$, if all $d_{i}$ 's are constant values, then we directly evaluate $B\left[x_{i} \leftarrow d_{i}\right]$ which is a constant truth value. If it is false, then we can exclude $c^{\prime}$ from $S C_{b, m s g}$. When some $d_{i}$ is a variable, we traverse backward in $c^{\prime}$ from the source state $s^{\prime}$ of the transition $t$ corresponding to $\mathrm{b} ! \mathrm{msg}\left(d_{1}, \ldots, d_{n}\right)$, and locate the first state $s \neq s^{\prime}$ such that $s$ has an incoming transition outside $c^{\prime}$ but within other cycles. If no such $s$ exists, then we take as $s$ the predecessor of $s^{\prime}$ in $c^{\prime}$. The path $p$ from $s$ to $s^{\prime}$ is then the longest acyclic path within $c$ that must be consecutively executed immediately before reaching the message sending statement. We compute the postcondition Post $(p)$ of $p$ by Floyd-Hoare-style forward inference starting with the precondition true ${ }^{2}$. This assumes that all the variables initially contain arbitrary values before $p$ is consecutively executed. If $\operatorname{Post}(p) \wedge B\left[x_{i} \leftarrow d_{i}\right]$ is unsatisfiable, then $c^{\prime}$ can be removed from $S C_{b, m s g}$. If the Promela model contains only linear arithmetic expressions in assignments and conditions, then the satisfiability of $\operatorname{Post}(p) \wedge B\left[x_{i} \leftarrow d_{i}\right]$ can be decided fully automatically using either an automated theorem prover or a linear programming solver. In the example in Figure 6 , we illustrate how to determine that $c_{3}$ cannot send a message to satisfy reply_status $=4$. The longest consecutively executed path $p$ in this example starts from the source state of the transition corresponding to the message receiving statement, i.e., the topmost state in the control flow graph of GIOPAgent. Then $\operatorname{Post}(p)=(\cdots \wedge(r s=5))$. Since $\operatorname{Post}(p) \wedge($ reply_status $=4)[$ reply_status $\leftarrow r s]$ is false, $c_{3}$ can be safely removed from $\left(c_{1}, S C_{\text {to ClientL, Reply }}\right)$.

\footnotetext{
${ }^{1} B\left[x_{i} \leftarrow d_{i}\right]$ is a boolean expression obtained from $B$ by substituting simultaneously each occurrence of $x_{i}$ with $d_{i}$.

${ }^{2}$ Since the path $p$ is acyclic, $\operatorname{Post}(p)$ can be computed fully automatically.
} 


\section{The Refinement of a Livelock Freedom Test}

We show how the discovery of cycle dependencies can be used to improve the precision of a livelock freedom test that we developed [12]. We sketch this test using the example in Figure 1.

In a Promela model we may label a set of statements as progress statements. Let us assume the message receiving statement (Line 6) in the process client is the only progress statement in Figure 1. A model is said to be free of livelock if and only if at least one of the progress statements must be repeated infinitely often in any infinite run of the model. Therefore, our example model is free of livelock if the client always receives replies from the server infinitely often. Moreover, we define a cycle to be a progress cycle if it contains at least one progress statement. So, the right cycle $c_{r}$ of client is the only progress cycle. We have shown in [12] that livelock freedom is undecidable for infinite state systems.

The basic idea of our livelock freedom test in [12] is to check whether there is any infinite run of a model in which no progress cycle is repeated infinitely often. If no such run exists, then the model is livelock free. In our test, we first abstract from arbitrary program code in the model and retain only the message sending and receiving statements. Next, we abstract from message orders and denote the message passing effect of a statement by an integer vector called an effect vector. Each component of an effect vector corresponds to one type of messages. A positive component represents the number of messages of the corresponding type being sent by the statement. A negative component represents the number of messages being received. We abstract further from the activation conditions and dependencies of cycles. The resulting abstract system is a set of cycles with their summary effect vectors. In our example, there are three cycles: $c_{l}$ with the effect vector $(1,0), c_{r}$ with $(0,-1)$, and $c_{s}$ with $(-1,1)$.

We now give a necessary condition for the existence of a livelocked run, i.e., a run in which no progress cycle is repeated infinitely often, in the form of an integer linear programming (ILP) problem. The ILP problem is shown in Figure 7. It can be solved in polynomial time. Intuitively, any solution to this ILP problem represents a combination of cycle effects that (1) can be repeated forever since it does not consume any type of messages (Inequalities 1-2); and (2) does not include any progress cycle (Inequality 3 ). The last inequality 4 restricts the number of times that a cycle is repeated to be non-negative. If the ILP problem has no solutions, then such cycle combination does not exist, which proves livelock freedom for the model. Unfortunately, the ILP problem has a solution: $x_{1}=1, x_{2}=x_{3}=0$. In this case, we do not know whether the model is livelock free or not because the abstraction used in our test is over-approximating and may introduce spurious behavior.

The above obtained ILP solution represents a counterexample suggesting the scenario that only the cycle $c_{l}$ is repeated infinitely often in some runs. However, by the help of our cycle dependency discovery, we can see that the cycle $c_{l}$ depends on $c_{r}$. Since $c_{r}$ is not included in the counterexample, the counterexample is spurious. Furthermore, we can use the cycle dependency to refine the 


$$
\begin{aligned}
\left(\begin{array}{l}
1 \\
0
\end{array}\right) x_{1}+\left(\begin{array}{c}
0 \\
-1
\end{array}\right) x_{2}+\left(\begin{array}{c}
-1 \\
1
\end{array}\right) x_{3} & \geq\left(\begin{array}{l}
0 \\
0
\end{array}\right) \\
x_{1}+x_{2}+x_{3} & >0 \\
x_{1} & =0 \\
x_{i} & \geq 0
\end{aligned}
$$

Fig. 7. The livelock freedom determination ILP problem for the model in Figure 1

abstraction by adding the following inequality to the ILP problem in Figure 7: $x_{1} \leq 3 x_{2}$. Intuitively, the new constraint says that the cycle $c_{r}$ must be executed at least once for every 3 times that $c_{l}$ is repeated. The determination of this constraint also relies on the estimation of the maximal iteration counts of the cycle $c_{l}$, which we discussed in [14].

The quality of the above described refinement procedure largely relies on the accuracy of the cycle dependency discovery techniques. The smaller a detected cycle dependency is, the more spurious behavior can be excluded through refinement.

\section{$7 \quad$ Experimental Results}

We have implemented different strategies to detect both local and global cycle dependencies for the models ${ }^{3}$ listed in Table 1 . The experimental results were obtained on a Pentium IV $1.60 \mathrm{GHz}$ machine with $1 \mathrm{~GB}$ memory.

Table 1. Test models

\begin{tabular}{lrr}
\hline Model & \# cycles & \# detected cycle dependencies \\
\hline i-Protocol & 22 & 30 \\
MVCC & 30 & 51 \\
GIOP & 66 & 203 \\
SMCS & 171 & 541 \\
\hline
\end{tabular}

We detected three types of dependencies: (1) dependencies on neighbors (see Corollary 1); (2) dependencies on cycles that may render the considered condition to be re-satisfied; (3) dependencies caused by message receiving statements. Table 1 lists the total number of dependencies of all three types that were detected for each model. Different strategies are used to over-approximate dependencies of Type 2 and 3, and their performances are compared as explained below.

${ }^{3}$ MVCC [8] models the Model View and Concurrent Control protocol used in the Clock toolkit for the development of groupware applications; $i$-Protocol [6] models a sliding-window protocol for Unix-to-Unix-Copy; GIOP [10] models inter-ORB message exchange and server object migration in the CORBA architecture; SMCS [17] models the T.122 and T.125 multi-point communication service protocol. 
Table 2. The comparison of different strategies to detect dependencies of Type 2. By the size of a cycle dependency $(c, S)$, we refer to the size of the set $S$. For each model and each strategy, we list the sum of the sizes of all the detected dependencies of this type.

\begin{tabular}{l|lll|ll|lll}
\hline & \multicolumn{3}{|l|}{ summary size of dependencies } & \multicolumn{2}{c|}{$\begin{array}{c}\text { \% reduction } \\
\text { w.r.t. MC }\end{array}$} & \multicolumn{3}{|c}{ runtime (secs.) } \\
\cline { 2 - 10 } Model & MC & ND & PC & ND & PC & MC & ND & PC \\
\hline i-Protocol & 63 & 63 & 43 & 0 & 31.7 & 13.02 & 13.27 & 13.66 \\
MVCC & 60 & 59 & 41 & 1.7 & 31.7 & 3.55 & 3.59 & 3.52 \\
GIOP & 837 & 788 & 714 & 5.9 & 14.7 & 29.25 & 30.12 & 33.17 \\
SMCS & 5200 & 5200 & 3424 & 0 & 34.2 & 136.63 & 143.75 & 175.05 \\
\hline
\end{tabular}

Table 7 compares three different strategies for the detection of dependencies of type 2: $M C$ is the coarsest one that includes a cycle in the dependency as long as it may influence at least one variable in the considered condition; $N D$ is the next-door strategy; $P C$ is the finest one that collects only preemptive cycles for computing dependencies. We observe that ND leads to only a minor improvement of the accuracy of the detected dependencies. PC reduces the sizes of dependencies much more effectively at the expense of a modest or even no runtime penalty (see the results for $\mathrm{MVCC}^{4}$ ). In particular, $\mathrm{PC}$ reduces the sizes of dependencies by more than one third for the model SMCS while ND does not reduce the cycle number at all.

Table 3. The comparison of different strategies to detect global dependencies caused by message receiving statements

\begin{tabular}{l|ll|l|ll}
\hline & \multicolumn{2}{|c|}{ summary size of dependencies } & $\begin{array}{r}\text { \% reduction } \\
\text { w.r.t. SC }\end{array}$ & \multicolumn{2}{r}{ runtime (secs.) } \\
\cline { 2 - 6 } Model & SC & FC & FC & SC & FC \\
\hline i-Protocol & 62 & 33 & 46.8 & 0.01 & 0.05 \\
MVCC & 35 & 35 & 0 & 0.01 & 0.66 \\
GIOP & 274 & 242 & 11.7 & 0.10 & 10.78 \\
SMCS & 410 & 338 & 17.5 & 1.37 & 45.17 \\
\hline
\end{tabular}

Table 3 shows two strategies to detect global dependencies caused by message receiving statements: $S C$-is coarser and includes any cycle in the dependency as long as it may send the same type of messages as received by the considered receiving statement. $F C$ checks the fastened cycles property in order to exclude cycles that cannot contribute a desired message. We observe that FC can reduce the sizes of dependencies quite considerably at the expense of a moderate to significant runtime penalty. The fact that FC did not reduce the dependency sizes for MVCC is expected because few variables are used in the model to store components of incoming messages. Moreover, those component storing variables are not used to control the behavior of the model, i.e., there are no message determined conditions. The extra runtime required by FC on MVCC was spent

\footnotetext{
${ }^{4}$ The reason is that ND and PC sometimes check only a small number of cycles for computing dependencies for one cycle while MC has to check all the cycles in the same process.
} 
on checking the existence of message determined conditions. If we know a priori that no such conditions exist in a model, which can be achieved by a manual scan of the Promela code, then FC is unnecessary.

To illustrate the benefit of our analysis we applied our approach to the counterexample refinement of our livelock freedom analysis for Promela models [12]. We have mentioned that, by obtaining smaller and more dependencies, we stand a better chance to determine spuriousness for the counterexample. The previous version of our prototype livelock freedom checker aLive used the ND strategy to discover local dependencies and was not able to detect global dependencies. In [12] we reported that the local cycle dependency detection helped to remove 7 counterexamples for a model of the Group Address Registration protocol for which livelock freedom was successfully proved. For the GIOP model, 8 counterexamples were found and aLive failed to prove spuriousness for one of them. The spuriousness of this counterexample is caused by abstracting away a global dependency. After we employed the FC strategy proposed in this paper in aLive, the one remaining counterexample in GIOP was determined to be spurious and subsequently excluded from the abstraction. The same was observed during the checking of the i-Protocol model for which 4 more spurious counterexamples were discovered due to the detection of global dependencies.

We also performed experiments in which we used the cycle dependency analysis in the spuriousness determination of counterexamples found during our buffer boundedness analysis [13]. The increase in precision that we achieved lies within the range of increase that we obtained for the livelock freedom analysis.

\section{Conclusion}

The first contribution of our work is a formalization of the concept of control flow cycle dependencies. The second contribution is that we presented several incomplete but efficient static analysis methods for the detection of both local and global cycle dependencies for reactive systems of concurrent processes. Furthermore, we conducted experiments that show the precision of this analysis when applied to a set of models of real-life systems. We also show that the precision of our approach compared to naive cycle dependency detection techniques improves the precision of our livelock freedom and buffer boundedness analyses since more spurious counterexamples can be detected.

Future work will include improving our analysis by incorporating data flow analysis. As an example, consider the computation of $E_{c}(B)$ as the set of variables that may influence the run-time values of the variables in $B$ along the cycle $c$ (Sec. 4.1). If a variable in $E_{c}(B)$ does not occur in $B$, then it must appear in the right hand side of an assignment statement that directly or indirectly changes the value of some variable $v$ in $B$. However, the effect of such an assignment may be killed later by an assignment to $v$ before the condition statement $(B)$ is reached. Therefore, the use of reachable definition analysis may improve the precision of $E_{c}(B)$. We will also consider broadening the approach to a wider range of programming and modeling languages. Finally, we see a potential for 
the application of cycle dependency analyses to other application areas, such as the prediction of temporal conflicts and spatial localities of code blocks for the improvement of instruction cache hit rates [11].

Acknowledgment. The work of the second author was supported by the DFGfunded research project IMCOS (Grant No. LE 1342/1-/2). We thank Daniel Butnaru for his assistance in programming the implementation prototype. Finally, we thank the anonymous referees for their valuable suggestions.

\section{References}

1. Bradley, A.R., Manna, Z., Sipma, H.B.: Termination of polynomial programs. In: Cousot, R. (ed.) VMCAI 2005. LNCS, vol. 3385, pp. 113-129. Springer, Heidelberg (2005)

2. Brand, D., Zafiropulo, P.: On communicating finite-state machines. Journal of the ACM 30(2), 323-342 (1983)

3. Cook, B., Podelski, A., Rybalchenko, A.: Abstraction refinement for termination. In: Hankin, C., Siveroni, I. (eds.) SAS 2005. LNCS, vol. 3672, pp. 87-101. Springer, Heidelberg (2005)

4. Corbett, J.C., Avrunin, G.S.: Using integer programming to verify general safety and liveness properties. Formal Methods in System Design 6(1), 97-123 (1995)

5. Cousot, P.: Proving program invariance and termination by parametric abstraction, lagrangian relaxation and semidefinite programming. In: Cousot, R. (ed.) VMCAI 2005. LNCS, vol. 3385, pp. 1-24. Springer, Heidelberg (2005)

6. Dong, Y., Du, X., Holzmann, G.J., Smolka, S.A.: Fighting livelock in the GNU i-Protocol: a case study in explicit-state model checking. Int. Journal on Software Tools for Technology Transfer (STTT) 4(4), 505-528 (2003)

7. Dwyer, M.B., Hatcliff, J.: Slicing software for model construction. In: Proc. of PEPM 1999, pp. 105-118 (1999)

8. Graham, T.C.N., Unnes, T., Nejabi, R.: Efficient distributed implementation of semi-replicated synchronous groupware. In: ACM Symposium on User Interface Software and Technology, pp. 1-10 (1996)

9. Holzmann, G.J.: The SPIN model checker: Primer and reference manual. Addison Wesley, Reading (2004)

10. Kamel, M., Leue, S.: Formalization and validation of the general Inter-ORB protocol (GIOP) using PROMELA and SPIN. Int. Journal on Software Tools for Technology Transfer (STTT) 2(4), 394-409 (2000)

11. Kumar, R., Tullsen, D.: Compiling for instruction cache performance on a multithreaded architecture. In: Proc. of MICRO 2002, pp. 419-429. ACM/IEEE (2002)

12. Leue, S., Ştefănescu, A., Wei, W.: A livelock freedom analysis for infinite state asynchronous reactive systems. In: Baier, C., Hermanns, H. (eds.) CONCUR 2006. LNCS, vol. 4137, pp. 79-94. Springer, Heidelberg (2006)

13. Leue, S., Mayr, R., Wei, W.: A scalable incomplete test for the boundedness of UML RT models. In: Jensen, K., Podelski, A. (eds.) TACAS 2004. LNCS, vol. 2988, pp. 327-341. Springer, Heidelberg (2004)

14. Leue, S., Wei, W.: Counterexample-based refinement for a boundedness test for CFSM languages. In: Godefroid, P. (ed.) SPIN 2005. LNCS, vol. 3639, pp. 58-74. Springer, Heidelberg (2005) 
15. Manna, Z., Pnueli, A.: The Temporal Logic of Reactive and Concurrent Systems - Specification. Springer, Heidelberg (1992)

16. Masticola, S.P., Ryder, B.G.: Non-concurrency analysis. In: PPOPP 1993, pp. 129138. ACM Press, New York (1993)

17. Merino, P., Troya, J.M.: Modeling and verification of the ITU-T multipoint communication service with SPIN. In: Proc. of SPIN 1996 (1996)

18. Millett, L.I., Teitelbaum, T.: Issues in slicing Promela and its applications to model checking, protocol understanding, and simulation. STTT 2(4), 343-349 (2000)

19. Naumovich, G., Avrunin, G.S.: A conservative data flow algorithm for detecting all pairs of statements that may happen in parallel. In: SIGSOFT FSE 1998, pp. 24-34. ACM Press, New York (1998)

20. Nielson, F., Nielson, H.R., Hankin, C.: Principles of program analysis, 2nd edn. Springer, Heidelberg (2005)

21. Pelánek, R.: BEEM: Benchmarks for explicit model checkers. In: Bošnački, D., Edelkamp, S. (eds.) SPIN 2007. LNCS, vol. 4595, pp. 263-267. Springer, Heidelberg (2007)

22. Podelski, A., Rybalchenko, A.: A complete method for the synthesis of linear ranking functions. In: Steffen, B., Levi, G. (eds.) VMCAI 2004. LNCS, vol. 2937, pp. 239-251. Springer, Heidelberg (2004)

23. Ranganath, V.P., Amtoft, T., Banerjee, A., Hatcliff, J., Dwyer, M.B.: A new foundation for control dependence and slicing for modern program structures. ACM Trans. Program. Lang. Syst. 29(5) (2007)

24. Siegel, S.F., Avrunin, G.S.: Improving the precision of INCA by eliminating solutions with spurious cycles. IEEE Trans. Software Eng. 28(2), 115-128 (2002)

25. Tip, F.: A survey of program slicing techniques. Journal of Programming Languages $3(3), 121-189$ (1995)

\section{A Appendix}

\section{A.1 The Proof of Theorem 1}

We prove the theorem by a reduction from the following undecidable problem [2]: the executability of a message reception in a system of communicating finite state machines (CFSM) ${ }^{5}$.

Instance: A CFSM $M$ and a local state $s$ of $M$ having an outgoing transition $t$ labeled by the receive action ' $? a$ '

Question: Does there exist a run of $M$ such that the message reception '? $a$ ' is executed at $s$ ?

We construct a CFSM system $M^{\prime}$ from $M$ by (1) introducing a new state $s^{\prime}$ in the same state machine as $s$ is; (2) adding at $s^{\prime}$ a self-transition labeled with ' $b$ ' where $b$ is a newly introduced type of message; (3) changing the target state of the transition $t$ to the newly introduced state $s^{\prime}$; and finally (4) adding a new state machine consisting of a single state $s^{\prime \prime}$ and a self-transition at $s^{\prime \prime}$.

\footnotetext{
${ }^{5}$ The proof is actually for the undecidability of the same problem for communicating finite state machines (CFSM). However, Promela models with unbounded buffers can simulate CFSM systems. Thus, the undecidability result also holds for Promela models.
} 
Moreover, let $c$ be the self-loop at $s^{\prime}$ and $S$ be the singleton cycle set consisting of the self-loop at $s^{\prime \prime}$.

We prove that '? $a$ ' can be executed at $s$ in $M$ if and only if $c$ does not depends on $S$ in $M^{\prime}$.

For the "if" part, assume that $c$ does not depends on $S$. Then, there exists an infinite run of $M^{\prime}$ in which $c$ is executed infinitely often while the self-loop at $s^{\prime \prime}$ ' is not. From the construction of $M^{\prime}, c$ can be executed only if '? $a$ ' can be executed at $s$ in $M^{\prime}$, which means that '? $a$ ' can be executed also in $M$.

For the "only if" part, assume that '? $a$ ' can be executed at $s$ in $M$. Then, '? $a$ ' can be also executed in $M^{\prime}$. After ' $? a$ ' is executed, $c$ can be repeated alone forever, which means $c$ does not depend on any other cycles for an infinite number of executions.

\section{A.2 The Proof of Proposition 1}

If $c$ is repeated an infinite number of times, then some other cycle $c^{\prime}$ in $p$ must be repeated also infinitely often. On every path from a state in $c$ to a state in $c^{\prime}$, there must be a transition $t$ from an exit state of $c$ such that $t$ is not contained in $c$. There are only finitely many such transitions, so one of them is taken an infinite number of times and it belongs to at least one of the neighbors of $c$.

\section{A.3 The Termination and Soundness of the Algorithm in Figure 4.1}

Proposition 3 (Termination). The algorithm in Figure 4.1 always terminates.

Proof. It is easy to see that no cycle can be added to visited more than once. Hence, each call to search_for_preemptive_cycles results in a new cycle being added to visited (Line 9). Note that our algorithm never removes any cycle from visited. Since there are only finitely many cycles, the algorithm must terminate.

Proposition 4 (Soundness). Given as an input a cycle $c$ in process $p$ such that $c$ is terminating on a locally determined condition $B$, the algorithm in Figure 4.1 returns a cycle dependency $(c, S)$ such that a cycle $c^{\prime} \in M C_{p}\left(E_{c}(B)\right)$ is preemptive if and only if $c^{\prime} \in S$.

Proof. We assign a natural number level $(d)$ to each cycle $d$ that is added to visited as follow: (1) level $(c)=0 ;(2)$ if $c_{1}$ is enqueued (Line 18) or added to ccs (Line 16) inside the call to search_for_preemptive_cycles $\left(c_{2}, B\right)$ and level $\left(c_{2}\right)=n$, then level $\left(c_{1}\right)=n+1$. In the second case, we say that $c_{2}$ is the parent of $c_{1}$ and $c_{1}$ is a child of $c_{2}$. Then, we can build a parent-child tree (PCT).

For the "if" part, we prove that if $c^{\prime} \in S$ then it is preemptive. It is easy to see that, in the path from the root $c$ to $c^{\prime}$ in the PCT, no cycle except $c$ and $c^{\prime}$ modifies any variable in $E_{c}(B)$. From this path, we can easily construct an acyclic path $\theta$ from an exit state $t$ in $c$ to an exit state $t^{\prime}$ in $c^{\prime}$, and an acyclic path $\theta^{\prime}$ from $t^{\prime}$ to $t$. $\theta$ and $\theta^{\prime}$ apparently do not modify any variable in $E_{c}(B)$.

For the "only if" part, assume that $c^{\prime}$ is preemptive. Then, there is an exit state $t$ in $c$, an exit state $t^{\prime}$ in $c^{\prime}$, an acyclic path $\theta$ from $t$ to $t^{\prime}$, and an acyclic 
path $\theta^{\prime}$ from $t^{\prime}$ to $t$ such that $\theta$ and $\theta^{\prime}$ do not modify any variable in $E_{c}(B)$. The path $\left\langle\theta, \theta^{\prime}\right\rangle$ can be decomposed into a set of cycles, from which we can construct a sequence of pairwise distinct cycles $c_{1}, \ldots, c_{n}$ such that (1) $c_{1}$ is a neighbor of $c,(2) c_{n}$ is a neighbor of $c^{\prime}$, and (3) each $c_{i}$ and $c_{i+1}$ are neighbors. It is easy to see no cycle in such a sequence modifies any variable in $E_{c}(B)$. Let $S E Q$ be the set of shortest sequences of cycles as constructed in this way. Assume that the sequences in $S E Q$ are of length $n$. For each sequence in $S E Q$, we add $c$ to its head and attach $c^{\prime}$ to the end. We prove that there is one sequence $s e q \in S E Q$ that is a path in the PCT, which implies that $c^{\prime}$ is added to $c c s$. The proof is by showing that, for any $k \leq n$, there is a sequence $s e q \in S E Q$ such that its prefix of length $i$ is a path in the PCT $\left(^{*}\right)$, by induction on the length $i$ of prefixes of sequences in $S E Q$.

Induction base: The prefix of length 1 of any sequence in SEQ is $c$, which is a path in the PCT.

Induction step: Assume that $\left(^{*}\right)$ holds for $k$. Let $P$ be the set of sequences in SEQ such that their prefixes of length $k$ are paths in the PCT. Let $C_{k}$ be the set of cycles $\{d \mid d$ is the $k$-th element in a sequence in $P\}$, and $C_{k+1}$ be $\{d \mid d$ is the $(k+1)$-th element in a sequence in $P\}$. By contradiction, we assume that there is no sequence in $P$ such that its prefix of length $(k+1)$ is a path in the PCT. Then, inside the call to search_for_preemptive_cycles $\left(c_{k}, B\right)$ for each $c_{k} \in C_{k}$, none of the neighbors of $c_{k+1}$ in $C_{k+1}$ is enqueued or added to visited. This happens only when $c_{k+1}$ is already in open or in visited. Let $p$ be the parent of $c_{k+1}$. So, $p \notin P$. We have either that (1) $\operatorname{level}(p)=k$, or that (2) $\operatorname{level}(p)<k$. When $\operatorname{level}(p)=k$, the path from $c$ to $p$ must be the prefix of length $k$ of some sequence in $S E Q$, which means that $p \in P$. This leads to a contradiction. When level $(p)<k$, we construct a sequence of cycles from any sequence in $P$ whose $(k+1)$-th element is $c_{k+1}$, by replacing the prefix of length $k$ by $p$. The new sequence is shorter than the sequences in SEQ, which contradicts that SEQ contains the shortest sequences of pairwise distinct cycles connecting $c$ and $c^{\prime}$.

\section{A.4 The Proof of Proposition 2}

Lemma 1. Using the notation in the definition of the fastened cycles property in Section 5, the following is satisfied: For any path $p_{1}$ that ends at an exit state $s$ within $p$, the path $p_{2}$ in $c$ from the source state of $t_{1}$ to $s$ is consecutively executed ${ }^{6}$ in the end of $p_{1}$.

Proof. We suppose that there are $q$ exit states in $p: e s_{1}, \ldots, e s_{q}$. We prove the lemma by induction on the index $k$ of $e s_{k}$.

Induction base: $e s_{1}$ is the source state of $t_{1}$. The path from $e s_{1}$ to $e s_{1}$ is an empty path which is always consecutively executed.

\footnotetext{
${ }^{6}$ Given two paths $p_{1}$ and $p_{2}$, we say that $p_{2}$ is executed in $p_{1}$ if $p_{2}$ is a subsequence of $p_{1}$. If $p_{2}$ is a consecutive subsequence of $p_{1}$, then we say that it is consecutively executed in $p_{1}$. In particular, an empty path is always consecutively executed.
} 
Induction step: Assume the lemma holds for $e s_{m}$ where $m<k$. Let $p^{\prime}$ denote the path from the source state of $t_{1}$ to $e s_{k}$. Suppose that $e s_{j}$ is the last exit state at which the execution of $p^{\prime}$ is interrupted. We have that $j<k$. From the induction assumption, immediately before the execution $p^{\prime}$ is resumed at $e s_{j}$, the path from the source state of $t_{1}$ to $e s_{j}$ is consecutively executed. Furthermore, after the execution of $p^{\prime}$ is resumed, the remaining part of $p^{\prime}$ is also consecutively executed. So, $p^{\prime}$ is consecutively executed.

In the following, we prove Proposition 2 using the above lemma.

Proof. We denote by $s_{1}$ the statement b?msg $\left(x_{1}, \ldots, x_{n}\right)$, by $s_{2}$ the statement $(B)$, by $t_{1}$ the transition corresponding to $s_{1}$, by $t_{2}$ the transition corresponding to $s_{2}$, and by $p$ the path from the source state of $t_{1}$ to the source state of $t_{2}$.

We denote by $s_{l}$ the exit state within $p$ at which the execution of $c$ is interrupted at the last time in a run. We denote by $p^{\prime}$ the path from the source state of $t_{1}$ to $s_{l}$ in $c$, and by $p^{\prime \prime}$ the path from $s_{l}$ to the source state of $t_{2}$ in $c$. So, $p=\left\langle p^{\prime}, p^{\prime \prime}\right\rangle$. Following Lemma 1 , before the execution of $c$ is resumed, $p^{\prime}$ is consecutively executed. Because $s_{l}$ is the last state at which $c$ is exited, $p^{\prime \prime}$ is also consecutively executed after $c$ is re-entered. So, $p$ is consecutively executed before the condition statement $s_{2}$ is reached. In this consecutive execution of $p$ a message $\operatorname{msg}\left(d_{1}, \ldots, d_{n}\right)$ is received and each variable $x_{i} \in \operatorname{var}(B)$ is assigned with $d_{i}$. After $p$ is executed, the execution of $c$ can continue if and only if $B$ is true. Since any variable $x_{i} \in \operatorname{var}(B)$ is not modified in $p$ after receiving the message, we have that $B\left[x_{i} \leftarrow d_{i}\right]$ is true. 